\title{
COINCIDENCE THEOREMS AND MATCHING THEOREMS
}

\author{
HWEI-MEI KO AND KOK-IKEONG TAN $\left.{ }^{*}\right)$
}

\begin{abstract}
Two coincidence theorems of Ky Fan are first slightly generalized. As applications, new matching theorems are obtained, one of which has several equivalent forms, including the classical KnasterKuratowski-Mazurkiewicz theorem.
\end{abstract}

\section{Introduction}

For a non-empty set $X$, we shall denote by $2^{X}$ the collection of all nonempty subsets of $X$. If $X$ is a topological space and $A \subset X$, we shall denote by $\bar{A}$ the closure of $A$ and by $\partial A$ the boundary of $A$. If $E$ is a topological vector space, we shall denote by $E^{\prime}$ the vector space of all continuous linear functionals on $E$ and by $\langle w, x\rangle$ for $w \in E^{\prime}$ and $x \in E$ the pairing between $E^{\prime}$ and $E$. If $A \subset E, \operatorname{co}(A)$ (respectively, $\overline{c o}(A)$ ) denotes the convex (respectively, closed convex) hull of $A$. Suppose $X \subset E$ is non-empty; then a map $f: X \rightarrow 2^{E}$ is said to be upper hemi-continuous ([1, p.68]; see also [2, p.133]) if for each $\phi \in E^{\prime}$ and for each real number $\lambda$, the set $\{x \in X: \sup \operatorname{Re}\langle\phi, u\rangle<\lambda\}$ is open in $X$. $u \in f(x)$

We note that every upper semi-continuous map is upper hemi-continuous and the sum of two upper hemi-continuous maps is again upper hemi-continuous.

Received August 13, 1990.

(*) This work was partially supported by NSERC of Canada under grant number A-8096. 1980 Mathematics Subject Classification (1985 Revision), 47H10, 54 H25.

Key words and phrases. Coincidence theorem, matching theorem, upper hemi-continuous, upper semi-continuous, upper demi-continuous, continuous linear functional, locally convex space, paracompact, compact convex, fixed point theorem, locally finite family. 
For relations among upper semi-continuity, upper demi-continuity [4] and upper hemi-continuity, we refer to Shih-Tan [11, Propositions 1 and 2 and Examples 1 and 2].

In this paper, we first slightly generalize coincidence theorems of Fan [5, Theorems 9 and 10] and a fixed point theorem of Shin-Tan [11, Theorem 4]. As applications, new matching theorems are obtained, some of which generalize those of Fan [5, Theorems 2 and 11]. Finally, from one of our matching theorems, we deduce several equivalent results, one of which is equivalent to the classical Knaster-Kuratowski-Mazurkiewicz theorem [9].

\section{Coincidence Theorems}

First we shall state without proof the following result which can be proved by slightly modifying the proofs of Theorem 9 of Fan in [5] and Lemma 1.2 of Ko-Tan in [10]:

Theorem 1. Let $X$ be a paracompact convex set in a locally convex Hausdorff topological vector space $E, X_{0}$ be a non-empty compact convex subset of $X$ and $K$ be a non-empty compact subset of $X$. Let $f, g: X \rightarrow 2^{E}$ be upper hemi-continuous such that

(a) For each $x \in X, f(x)$ and $g(x)$ are closed convex, at least one of which is compact.

(b) For any $x \in K \cap \partial X$ and $\phi \in E^{\prime}$ with $\operatorname{Re} \phi(x) \leq \operatorname{Re} \phi(y)$ for all $y \in X$, there exist $u \in f(x)$ and $v \in g(x)$ such that $\operatorname{Re} \phi(u) \geq \operatorname{Re} \phi(v)$.

(c) For any $x \in X \backslash K$ and $\phi \in E^{\prime}$ with $\operatorname{Re} \phi(x) \leq \operatorname{Re} \phi(y)$ for all $y \in X_{0}$, there exist $u \in f(x)$ and $v \in g(x)$ such that $\operatorname{Re} \phi(u) \geq \operatorname{Re} \phi(v)$.

Then there exists a point $\hat{x} \in X$ such that $f(\hat{x}) \cap g(\hat{x}) \neq \phi$.

The following result is a consequence of Theorem 1 and is a generalization of Theorem 10 of Fan in [5] and Theorem 4 of Shih-Tan in [11]:

Theorem 2. Let $X$ be a paracompact convex set in a locally convex Haus- 
dorff topological vector space $E, X_{0}$ be a non-empty compact convex subset of $X$ and $K$ be a non-empty compact subset of $X$. Let $f, g: X \rightarrow 2^{E}$ be upper hemi-continuous such that

(a) For each $x \in X, f(x)$ and $g(x)$ are closed convex at least one of which is compact.

(b) For each $x \in K \cap \partial X, f(x)-g(x)$ meets $\overline{\bigcup_{\lambda>0} \lambda(X-x)}$.

(c) For each $x \in X \backslash K, f(x)-g(x)$ meets $\overline{\bigcup_{\lambda>0} \lambda\left(X_{0}-x\right)}$.

Then there exists a point $\hat{x} \in X$ such that $f(\hat{x}) \cap g(\hat{x}) \neq \phi$.

Proof. Let $x \in K \cap \partial X$ and $\phi \in E^{\prime}$ be such that

$$
\operatorname{Re} \phi(x) \leq \operatorname{Re} \phi(y) \text { for all } y \in X
$$

As $f(x)-g(x)$ meets $\overline{\bigcup_{\lambda>0} \lambda(X-x)}$, let $u \in f(x), v \in g(x),\left(\lambda_{\alpha}\right)_{\alpha \in \Gamma}$ be a net in $(0, \infty)$ and $\left(x_{\alpha}\right)_{\alpha \in \Gamma}$ be a net in $X$ such that $\lambda_{\alpha}\left(x_{\alpha}-x\right) \rightarrow u-v$; it follows that

$$
\lambda_{\alpha} \phi\left(x_{\alpha}-x\right)=\phi\left(\lambda_{\alpha}\left(x_{\alpha}-x\right)\right) \rightarrow \phi(u-v)=\phi(u)-\phi(v) .
$$

By (1), for each $\alpha \in \Gamma, \operatorname{Re} \phi(x) \leq \operatorname{Re} \phi\left(x_{\alpha}\right)$, so that $\operatorname{Re} \phi(u) \geq \operatorname{Re} \phi(v)$. Thus the condition (b) in Theorem 1 is satisfied.

Next let $x \in X \backslash K$ and $\phi \in E^{\prime}$ be such that

$$
\operatorname{Re} \phi(x) \leq \operatorname{Re} \phi(y) \text { for all } y \in X_{0} .
$$

As $f(x)-g(x)$ meets $\overline{\bigcup_{\lambda>0} \lambda\left(X_{0}-x\right)}$, let $u \in f(x), v \in g(x),\left(\lambda_{\alpha}\right)_{\alpha \in \Gamma}$ be a net in $(0, \infty)$ and $\left(x_{\alpha}\right)_{\alpha \in \Gamma}$ be a net in $X_{0}$ such that $\lambda_{\alpha}\left(x_{\alpha}-x\right) \rightarrow u-v$; it follows from (2) that $\operatorname{Re} \phi(u) \geq \operatorname{Re} \phi(v)$. Thus the condition (c) in Theorem 1 is also satisfied.

Therefore by Theorem 1, there exists $\hat{x} \in X$ such that $f(\hat{x}) \cap g(\hat{x}) \neq \phi$.

We note that Theorem 2 remains valid if in the union $\bigcup_{\lambda>0}$ in both conditions (b) and (c) " $\lambda>0$ " is replaced by " $\lambda<0$ ". 
When $g(x)=x$ for all $x \in X$, the coincidence Theorem 2 becomes the following very general fixed point theorem which generalizes a fixed point theroem of Halpern [7, Theorem 2] which in turn generalizes Fan-Glicksberg's infinite dimensional generalization [3,6] of the Kakutani fixed point theorem [8]:

Theorem 3. Let $X$ be a paracompact convex set in a locally convex Hausdorff topological vector space $E, X_{0}$ be a non-empty compact convex subset of $X$ and $K$ be a non-empty compact subset of $X$. Let $f: X \rightarrow 2^{E}$ be an upper hemi-continuous map such that

(a) For each $x \in X, f(x)$ is closed and convex.

(b) For each $x \in K \cap \partial X, f(x) \bigcap\left[x+\overline{\bigcup_{\lambda>0} \lambda(X-x)}\right] \neq \phi$.

(c) For each $x \in X \backslash K, f(x) \cap\left[x+\overline{\bigcup_{\lambda>0} \lambda\left(X_{0}-x\right)}\right] \neq \phi$.

Then there exists a point $\hat{x} \in X$ such that $\hat{x} \in f(\hat{x})$.

As is noted earlier, Theorem 3 remains valid if in the union $\bigcup_{\lambda>0}$ in both conditions (b) and (c) " $\lambda>0$ " is replaced by " $\lambda<0$ " and the result so formulated generalizes Theorem 3 in [7].

\section{Matching Theorems}

As an application of Theorem 2, we have the following matching theorem for closed coverings of a convex set:

Theorem 4. Let $X$ be a paracompact convex subset of a locally convex Hausdorff topological vector space $E, X_{0}$ be a non-empty compact convex subset of $X$ and $K$ be a non-empty compact sulset of $X$. Let $\left\{A_{i}: i \in l\right\}$ and $\left\{B_{j}: j \in\right.$ $J\}$ be two locally finite families of closed subsets of $X$ such that

$$
\bigcup_{i \in l} A_{i}=\bigcup_{j \in J} B_{j}=X
$$

Let $\left\{C_{i}: i \in l\right\}$ and $\left\{D_{j}: j \in J\right\}$ be two families of non-empty subsets of $E$ such that any finite union of the $C_{i}$ 's is contained in a compact convex subset of 
$E$. Let $s: X \rightarrow 2^{E}$ be upper hemi-continuous such that each $s(x)$ is a compact convex set. Suppose that for each point $x \in(K \cap \partial X) \bigcup(X \backslash K)$, there exist $i \in l$ and $j \in J$ sucht that

(i) $x \in A_{i} \cap B_{j}$,

(ii) $\overline{c o}\left(C_{i}+s(x)\right)-\overline{c o}\left(D_{j}\right)$ meet $\begin{cases}\overline{\bigcup_{\lambda>0}(X-x)}, & \text { if } x \in K \cap \partial X, \\ \overline{\bigcup_{\lambda>0}\left(X_{0}-x\right)}, & \text { if } x \in X \backslash K .\end{cases}$

Then there exist two non-empty finite sets $l_{0} \subset l$ and $J_{0} \subset J$ and a point $\hat{x} \in X$ such that

(a) $\hat{x} \in\left(\bigcap_{i \in l_{0}} A_{i}\right) \cap\left(\bigcap_{j \in J_{0}} B_{j}\right)$,

(b) $\overline{c o}\left(\bigcup\left\{C_{i}: i \in l_{0}\right\}\right)+s(\hat{x})$ meets $\overline{c o}\left(\bigcup\left\{D_{j}: j \in J_{0}\right\}\right)$.

Proof. For each $x \in X$, let

$$
l(x)=\left\{i \in l: x \in A_{i}\right\}, J(x)=\left\{j \in J: x \in B_{j}\right\}
$$

then $l(x)$ and $J(x)$ are non-empty and finite as $\bigcup_{i \in l} A_{i}=\bigcup_{j \in J} B_{j}=X$ and $\left\{A_{i}: i \in l\right\}$ and $\left\{B_{j}: j \in J\right\}$ are locally finite. Define $f, g, h: X \rightarrow 2^{E}$ by

$$
\begin{aligned}
& f(x)=\overline{c o}\left(\cup\left\{C_{i}+s(x): i \in l(x)\right\}\right), \\
& g(x)=\overline{c o}\left(\cup\left\{D_{j}: j \in J(x)\right\}\right), \\
& h(x)=\overline{c o}\left(\cup\left\{C_{i}: i \in l(x)\right\}\right) .
\end{aligned}
$$

By hypotheses, for each $x \in X, h(x)$ and $s(x)$ are compact convex so that $f(x)=h(x)+s(x)$ is also compact convex. Since $\left\{A_{i}: i \in l\right\}$ is a locally finite family of closed subsets of $X$, for each $x \in X$, the set $U(x)=X \backslash \bigcup_{i \notin l(x)} A_{i}$ is an open neighborhood of $x$ in $X$; note then whenever $y \in U(x), y \notin A_{i}$ for each $i \notin l(x)$ so that $l(y) \subset l(x)$ and therefore $h(y) \subset h(x)$. This shows that $h$ is upper semi-continuous and hence $f=h+s$ is upper hemi-continuous. Similarly we can show that $g$ is upper semi-continuous (and hence upper hemi-continuous) on $X$. Thus the condition (a) of Theorem 2 is satisfied. By (i) and (ii), the conditions (b) and (c) of Theorem 2 are also satisfied. By Theorem 2, there exists $\hat{x} \in X$ 
such that $f(\hat{x}) \bigcap g(\hat{x}) \neq \phi$. Let $l_{0}=l(\hat{x})$ and $J_{0}=J(\hat{x})$, then $l_{0}$ and $J_{0}$ are non-empty and finite and the conclusions of the theorem hold.

The proof of the above theorem is a modification of Theorem 11 of Fan in [5] and of Theorem 1 of Shih-Tan in [11]. Theorem 4 generalizes Theorem 11 and hence also Theorem 12 of Fan in [5]. The following result is an easy consequence of Theroem 4:

Theorem 5. Let $X$ be a paracompact convex subset of a locally convex Hausdorff topological vector space $E, X_{0}$ be a non-empty compact convex subset of $X$ and $K$ be a non-empty compact subset of $X$. Let $\left\{A_{i}: i \in l\right\}$ and $\left\{B_{j}: j \in\right.$ $J\}$ be two locally finite families of closed subsets of $X$ such that

$$
\bigcup_{i \in l} A_{i}=\bigcup_{j \in J} B_{j}=X
$$

Let $\left\{C_{i}: i \in l\right\}$ and $\left\{D_{j}: j \in J\right\}$ be two families of non-empty subsets of $E$ such that any finite union of $C_{i}$ 's is contained in a compact convex subset of $E$. Suppose that for each point $x \in(K \cap \partial X) \cup(X \backslash K)$, there exist $i \in l$ and $j \in J$ such that

(i) $x \in A_{i} \cap B_{j}$,

(ii) $\overline{c o}\left(C_{i}\right)-\overline{c o}\left(D_{j}\right)$ meets $\begin{cases}x+\overline{\bigcup_{\lambda>0} \lambda(X-x)}, & \text { if } x \in K \cap \partial X, \\ x+\overline{\bigcup_{\lambda>0} \lambda\left(X_{0}-x\right)}, & \text { if } x \in X \backslash K .\end{cases}$ Then there exist non-empty finite sets $l_{0} \subset l$ and $J_{0} \subset J$ such that

$$
\left(\bigcap_{i \in l_{0}} A_{i}\right) \cap\left(\bigcap_{j \in J_{0}} B_{j}\right) \cap\left(\overline{c o}\left(\bigcup_{i \in l_{0}} C_{i}\right)-\overline{c o}\left(\bigcup_{j \in J_{0}} D_{j}\right)\right) \neq \phi
$$

Proof. Let $s: X \rightarrow 2^{E}$ be defined by $s(x)=-x$ for all $x \in X$. Then all hypotheses of Theorem 4 are satisfied so that there exist non-empty finite sets $l_{0} \subset l$ and $J_{0} \subset J$ and a point $\hat{x} \in X$ such that
(a) $\hat{x} \in\left(\bigcap_{i \in l_{0}} A_{i}\right) \cap\left(\bigcap_{j \in J_{0}} B_{j}\right)$,
(b) $\left(\overline{c o}\left(\bigcup_{i \in l_{0}} C_{i}\right)-\hat{x}\right) \cap \overline{c o}\left(\bigcup_{j \in J_{0}} D_{j}\right) \neq \phi$; 
it follows that

$$
\hat{x} \in\left(\bigcap_{i \in l_{0}} A_{i}\right) \cap\left(\bigcap_{j \in J_{0}} B_{j}\right) \cap\left(\overline{c o}\left(\bigcup_{i \in l_{0}} C_{i}\right)-\overline{c o}\left(\bigcup_{i \in J_{0}} D_{j}\right)\right) .
$$

As an application of Theorem 5 , we have

Theorem 6. Let $Y$ be a non-empty convex set in a Hausdorff topological

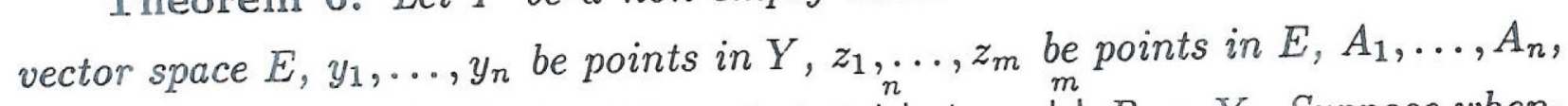
$B_{1}, \ldots, B_{m}$ be closed subsets of $Y$ such that $\bigcup_{i=1}^{n} A_{i}=\bigcup_{j=1}^{m} B_{j}=Y$. Suppose whenever $1 \leq i_{1}<\ldots<i_{k} \leq n$ with $1 \leq k<n$ and whenever $x \in \operatorname{co}\left\{y_{i_{1}}, \ldots, y_{i_{k}}\right\}$, there exists $j \in\{1, \ldots, m\}$ such that $x \in B_{j}$ and $x+z_{j} \in \operatorname{co}\left\{y_{i_{1}}, \ldots, y_{i_{k}}\right\}$. Then there exist two non-empty subfamilies $\mathcal{G}$ of $\{1, \ldots, n\}$ and $\mathcal{H}$ of $\{1, \ldots, m\}$ such that

$$
\left(\bigcap_{i \in \mathcal{G}} A_{i}\right) \cap\left(\bigcap_{j \in \mathcal{H}} B_{j}\right) \cap\left[\left(\operatorname{co}\left\{y_{i}: i \in \mathcal{G}\right\}\right)-\left(\operatorname{co}\left\{z_{j}: j \in \mathcal{H}\right\}\right)\right] \neq \phi
$$

Proof. Let $X=X_{0}=K=\operatorname{co}\left\{y_{1}, \ldots, y_{n}\right\}, l=\{1, \ldots, n\}, J=\{1, \ldots, m\}$ and

$$
\begin{aligned}
& C_{i}=\left\{y_{i}\right\}, \tilde{A}_{i}=A_{i} \cap X \quad \text { for each } i \in l, \\
& D_{j}=\left\{z_{j}\right\}, \tilde{B}_{j}=B_{j} \cap X \text { for each } j \in J,
\end{aligned}
$$

We shall show that for each $x \in \partial X$, there exist $i \in l$ and $j \in J$ such that

(i) $x \in \tilde{A}_{i} \cap \tilde{B}_{j}$,

(ii) $y_{i}-z_{j} \in x+\bigcup_{\lambda>1} \lambda(X-x)$.

Indeed, let $x \in \partial X$; without loss of generality we may assume that $x=\sum_{k=1}^{s} a_{k} y_{k}$ where $1 \leq s<n$ and $a_{k}>0$ for each $k=1, \ldots, s$ with $\sum_{k=1}^{s} a_{k}=1$. Thus by hypothesis, there exist $i \in l$ and $j \in J$ such that $x \in \tilde{A}_{i} \cap \tilde{B}_{j}$ and $x+z_{j} \in$ $\operatorname{co}\left\{y_{1}, \ldots, y_{s}\right\}$. Let $x+z_{j}=\sum_{k=1}^{s} b_{k} y_{k}$ where $b_{k} \geq 0$ for each $k=1, \ldots, s$ and $\sum_{k=1}^{s} b_{k}=1$. As $a_{k}>0$ for all $k=1, \ldots, s$, we can choose $\lambda>1$ such that $\lambda a_{k}-b_{k} \geq 0$ for all $k=1, \ldots, s$. As $\sum_{k=1}^{s}\left(\lambda a_{k}-b_{k}\right)=\lambda\left(\sum_{k=1}^{s} a_{k}\right)-\left(\sum_{k=1}^{s} b_{k}\right)=\lambda-1$, 
$\frac{1}{\lambda-1} \sum_{k=1}^{s}\left(\lambda a_{k}-b_{k}\right) y_{k}$ is in $X$. It follows that

$$
\begin{aligned}
& \frac{1}{\lambda}\left(y_{i}-z_{j}-x\right)+x=\frac{1}{\lambda}\left(y_{i}-\sum_{k=1}^{s} b_{k} y_{k}\right)+\left(\sum_{k=1}^{s} a_{k} y_{k}\right) \\
= & \frac{1}{\lambda}\left[1 \cdot y_{i}+(\lambda-1) \cdot \frac{1}{\lambda-1} \sum_{k=1}^{s}\left(\lambda a_{k}-b_{k}\right) y_{k}\right] \in X
\end{aligned}
$$

so that $y_{i}-z_{j} \in x+\lambda(X-x) \subset x+\bigcup_{\lambda>1} \lambda(X-x)$.

Since $X$ is a compact convex set in a Euclidean space, Theorem 5 implies there exists two non-empty subfamilies $\mathcal{G}$ of $\{1, \ldots, n\}$ and $\mathcal{H}$ of $\{1, \ldots, m\}$ such that

$$
\begin{aligned}
& \left(\bigcap_{i \in g} \tilde{A}_{i}\right) \cap\left(\bigcap_{j \in \mathcal{H}} \tilde{B}_{j}\right) \cap\left(\operatorname{co}\left\{y_{i}: i \in \mathcal{G}\right\}-\operatorname{co}\left\{z_{j}: j \in \mathcal{H}\right\}\right) \neq \phi \\
& \left(\bigcap_{i \in g} A_{i}\right) \cap\left(\bigcap_{j \in \mathcal{H}} B_{j}\right) \cap\left(\operatorname{co}\left\{y_{i}: i \in \mathcal{G}\right\}-\operatorname{co}\left\{z_{j}: j \in \mathcal{H}\right\}\right) \neq \phi .
\end{aligned}
$$

This completes the proof.

\section{Equivalent forms of the Classical $\mathbb{K}$ naster-Kuratowski-}

\section{Mazurkiewicz Theorem}

By taking $z_{j}=0$ and $B_{j}=Y$ for all $j=1, \ldots, m$ in Theorem 6 , we have the following very general matching theorem due to Fan [5, Theorem 2] where he has given two basically different proofs:

Theorem 7A. Let $Y$ be a non-empty convex set in a Hausdorff topological vector space $E, y_{1}, \ldots, y_{n}$ be points in $Y, A_{1}, \ldots, A_{n}$ be closed subsets of $Y$ such that $\bigcup_{i=1}^{n} A_{i}=Y$. Then there exists a non-empty subset $\left\{i_{1}, \ldots, i_{k}\right\}$ of $\{1, \ldots, n\}$ such that

$$
\left(\bigcap_{j=1}^{n} A_{i_{j}}\right) \cap \operatorname{co}\left\{y_{i_{j}}: j=1, \ldots, k\right\} \neq \phi .
$$

Now we shall state the following Theorems 7B, 7C, 7D and 7E which are all equivalent to Theorem $7 \mathrm{~A}$ : 
Theorem 7B. Let $Y$ be a non-empty convex set in a Hausdorff topological vector space $E, y_{1} \ldots, y_{n}$ be points in $Y$ and $B_{1}, \ldots, B_{n}$ be open subsets of $Y$ such that $\bigcup_{i=1}^{n} B_{i}=Y$. Then there exists a non-empty subset $\left\{i_{i}, \ldots, i_{k}\right\}$ of $\{1, \ldots, n\}$ such that

$$
\left(\bigcap_{j=1}^{k} B_{i_{j}}\right) \cap\left(\operatorname{co}\left\{y_{i_{j}}: j=1, \ldots, k\right\}\right) \neq \phi
$$

Theorem 7C. Let $Y$ be a non-empty convex set in a Hausdorff topological vector space $E, y_{1}, \ldots, y_{n}$ be points in $Y$ and $A_{1}, \ldots, A_{n}$ be closed subsets of $Y$ such that $\operatorname{co}\left\{y_{i}: i \in l\right\} \subset \bigcup_{i \in l} A_{i}$ for each subset l of $\{1, \ldots, n\}$. Then $\bigcap_{i=1}^{n} A_{i} \neq \phi$.

Theorem 7D. Let $Y$ be a non-empty convex set in a Hausdorff topological vector space $E, y_{1}, \ldots, y_{n}$ be points in $Y$ and $B_{1}, \ldots, B_{n}$ be open subsets of $Y$ such that $c\left\{\left\{y_{i}: i \in l\right\} \subset \bigcup_{i \in l} B_{i}\right.$ for each subset $l$ of $\{1, \ldots, n\}$. Then $\bigcap_{i=1}^{n} B_{i} \neq \phi$.

Theorem $7 \mathrm{E}$. Let $Y$ be a non-empty convex set in a Hausdorff topological vector space $E,\left\{y_{i}: i \in l\right\}$ be a family of points in $Y$ which is contained in a compact convex subset of $Y$ and $\left\{O_{i}: i \in l\right\}$ be a family of open subsets of $Y$ such that $\bigcup_{i \in I} O_{i}=Y$. Then there exists a non-empty finite subset $l_{0}$ of $l$ such that

$$
\left(\bigcap_{i \in l_{0}} O_{i}\right) \cap\left(\operatorname{co}\left\{y_{i}: i \in l_{0}\right\}\right) \neq \phi
$$

We first remark that Theorem $7 \mathrm{~A}$ and Theorem $7 \mathrm{~B}$ (respectively, Theorem $7 \mathrm{C}$ and Theorem 7D) are dual statements of each other in the sense that the words "closed" and "open" are interchangeable. Ilowever, a dual statement of Theorem $7 \mathrm{E}$ does not hold even if the set $Y$ is compact as the following simple example illustrates:

Example. Let $Y=l=[0,1]$. For each $i \in l$ with $i \neq \frac{1}{3}, \frac{1}{2}$, $\frac{2}{3}$, let $A_{i}=\{1-i\}$ and let $A_{\frac{1}{3}}=\left\{\frac{1}{2}\right\}, A_{\frac{1}{2}}=\left\{\frac{2}{3}\right\}, A_{\frac{2}{3}}=\left\{\frac{1}{3}\right\}$. Then $\left\{A_{i}: i \in l\right\}$ is a family of closed subsets of $Y$ such that

$$
\text { (a) } \bigcup_{i \in l} A_{i}=Y
$$


(b) $i \notin A_{i}$ for each $i \in l$;

(c) $A_{i} \cap A_{j}=\phi$ if $i \neq j$.

Thus there does not exist a non-empty finite subset $l_{0}$ of $l$ such that

$$
\left(\bigcap_{i \in l_{0}} A_{i}\right) \cap\left(\operatorname{co}\left\{i: i \in l_{0}\right\}\right) \neq \phi
$$

We next remark that Thoerem $7 \mathrm{C}$ is equivalent to the classical KnasterKuratowski-Mazurkiewicz theorem [9] while Theorem 7D is equivalent to Corollary 1 in [12]. However the formulations of Theorems $7 \mathrm{~B}$ and $7 \mathrm{E}$ appear to be new. Thus once we have established the equivalence of Theorems $7 \mathrm{~A}, 7 \mathrm{~B}, 7 \mathrm{C}$, $7 \mathrm{D}$ and $7 \mathrm{E}$, our matching Theorem 6 and hence also Theorems 4 and 5, are all generalizations of the classical Knaster-Kuratowski-Mazurkiewicz theorem.

It is easy to see that Theorems $7 \mathrm{~B}$ and $7 \mathrm{E}$ imply each other. Before we proceed to establish the equivalence of Theorems $7 \mathrm{~A}, 7 \mathrm{~B}, 7 \mathrm{C}$ and $7 \mathrm{D}$, we observe that by replacing $A_{i}$ 's and $B_{i}$ 's by $A_{i} \bigcap \operatorname{co}\left\{y_{1}, \ldots, y_{n}\right\}$ and $B_{i} \cap \operatorname{co}\left\{y_{1}, \ldots, y_{n}\right\}$ respectively, we may assume without loss of generality that $Y=\operatorname{co}\left\{y_{i}, \ldots, y_{n}\right\}$ and is therefore also compact in some Euclidean space. We shall only prove that Theorem $7 \mathrm{~A} \Leftrightarrow$ Theorem $7 \mathrm{~B}$ and Theorem $7 \mathrm{~B} \Leftrightarrow$ Theorem $7 \mathrm{C}$; the proof of Theorem $7 \mathrm{~A} \Leftrightarrow$ Theorem 7D follows a similar argument as that of Theorem $7 \mathrm{~B}$ $\Leftrightarrow$ Theorem $7 \mathrm{C}$ and is thus omitted.

\section{Proof of Theorem $7 \mathrm{~A} \Rightarrow$ Theorem $7 \mathrm{~B}$ :}

For each $z \in Y$, let $H_{z}=\bigcap\left\{B_{i}: i=1, \ldots, n\right.$ and $\left.z \in B_{i}\right\}$; then $H_{z}$ is an open neighborhood of $z$ in $Y$ so that there exists an open neighborhood $U_{z}$ of $z$ in $Y$ such that $U_{z} \subset \bar{U}_{z} \subset H_{z}$. Since $Y=\bigcup\left\{U_{z}: z \in Y\right\}$ and $Y$ is compact (remember, we have assumed $Y=\operatorname{co}\left\{y_{1}, \ldots, y_{n}\right\}$, see remark above.) there exist $z_{1}, \ldots, z_{m}$ in $Y$ such that $Y=\bigcup\left\{U_{z}, j=1, \ldots, m\right\}$. For each $i=1, \ldots, n$, define

$$
A_{i}=\bigcup\left(\bar{U}_{z}, j=1, \ldots, m \text { and } H_{z_{j}} \subset B_{i}\right\}
$$

then $A_{i}$ is a closed subset of $Y$ and $A_{i} \subset B_{i}$. Clearly $Y=U_{i=1}^{n} A_{i}$. Hence by 
Theorem $7 \mathrm{~A}$, there exists a non-empty subset $\left\{i_{1}, \ldots, i_{k}\right\}$ of $\{1, \ldots, n\}$ such that

$$
\left(\bigcap_{j=1}^{k} A_{i_{j}}\right) \cap \operatorname{co}\left\{y_{i_{j}}: j=1, \ldots, k\right\} \neq \phi ;
$$

but then

$$
\left(\bigcap_{j=1}^{k} B_{i_{j}}\right) \cap \operatorname{co}\left\{y_{i_{j}}: j=1, \ldots, k\right\} \neq \phi .
$$

\section{Proof of Theorem 7B $\Rightarrow$ Theorem 7A:}

Fix any positive integer $m$. For each $i=1, \ldots, n$, let

$$
B_{i}(m)=\left\{y \in Y: \operatorname{dist}\left(y, A_{i}\right)<\frac{1}{m}\right\},
$$

(remember we have assumed $Y=c o\left\{y_{1}, \ldots, y_{n}\right\}$ so that $Y$ is contained in some Euclidean space.) then $B_{i}(m)$ is an open subset of $Y$ and $A_{i} \subset B_{i}(m)$. Since $\bigcup_{i=1}^{n} A_{i}=Y$, we have $\bigcup_{i=1}^{n} B_{i}(m)=Y$. Hence by Theorem $7 \mathrm{~B}$, there exists a non-empty subset $l_{m}$ of $\{1, \ldots, n\}$ such that

$$
\left(\bigcap_{i \in l_{m}} B_{i}(m)\right) \cap \operatorname{co}\left\{y_{i}: i \in l_{m}\right\} \neq \phi .
$$

Since the collection of all non-empty subsets of $\{1, \ldots, n\}$ is finite, there is a sequence $\left(m_{j}\right)_{j=1}^{\infty}$ with $m_{j} \rightarrow \infty$ as $j \rightarrow \infty$ and there is a non-empty subset $l_{0}$ of $\{1, \ldots, n\}$ such that $l m_{j}=l_{0}$ for all $j=1,2, \ldots$ For each $j=1,2, \ldots$, choose any $x_{j}$ in $\left(\bigcap_{i \in l_{0}} B_{i}\left(m_{j}\right)\right) \cap\left(c o\left\{y_{i}: i \in l_{0}\right\}\right)$. Since $\left(x_{j}\right)_{j=1}^{\infty}$ is a sequence in the compact set $\operatorname{co}\left\{y_{i}: i \in l_{0}\right\}$, there is a subsequence $\left(x_{l_{j}}\right)_{j=1}^{\infty}$ such that $x_{l_{j}} \rightarrow \hat{x}$ for some $\hat{x} \in \operatorname{co}\left\{y_{i}: i \in l_{0}\right\}$. Fix $i \in l_{0}$. Since

$$
\begin{aligned}
\operatorname{dist}\left(\hat{x}, A_{i}\right) & \leq \operatorname{dist}\left(\hat{x}, x_{l_{j}}\right)+\operatorname{dist}\left(x_{l_{j}}, A_{i}\right) \\
& <\operatorname{dist}\left(\hat{x}, x_{l_{j}}\right)+\frac{1}{m_{l_{j}}} \\
& \rightarrow 0 \text { as } j \rightarrow \infty,
\end{aligned}
$$

we see that $\hat{x} \in A_{i}$ as $A_{i}$ is closed. Therefore $\hat{x} \in \bigcap_{i \in l_{0}} A_{i}$ so that

$$
\left(\bigcap_{i \in l_{0}} A_{i}\right) \cap\left(\operatorname{co}\left\{y_{i}: i \in l_{0}\right\}\right) \neq \phi .
$$


Proof of Theorem 7B $\Rightarrow$ Theorem 7C:

Suppose $\bigcap_{i=1}^{n} A_{i}=\phi$; then $Y=Y \backslash \bigcap_{i=1}^{n} A_{i}=\bigcup_{i=1}^{n}\left(Y \backslash A_{i}\right)$. Let $B_{i}=Y \backslash A_{i}$ for each $i=1, \ldots, n$, then each $B_{i}$ is open in $Y$. By Theorem $7 \mathrm{~B}$, there exists a non-empty subset $\left\{i_{1}, \ldots, i_{k}\right\}$ of $\{1, \ldots, n\}$ such that

$$
\left(\bigcap_{j=1}^{k} B_{i_{j}}\right) \cap \operatorname{co}\left\{y_{i_{1}}, \ldots, y_{i_{k}}\right\} \neq \phi .
$$

Thus $\left(Y \backslash \bigcup_{j=1}^{k} A_{i_{j}}\right) \cap\left(\operatorname{co}\left\{y_{i_{1}}, \ldots, y_{i_{k}}\right\}\right) \neq \phi$ so that $\operatorname{co}\left\{y_{i_{1}}, \ldots, y_{i_{k}}\right\} \not \subset \bigcup_{j=1}^{k} A_{i_{j}}$ which is a contradiction. Therefore we must have

$$
\bigcap_{i=1}^{n} A_{i} \neq \phi \text {. }
$$

\section{Proof of Theorem 7C $\Rightarrow$ Theorem 7B:}

Suppose the contrary that for any non-empty subset $l$ of $\{1, \ldots, n\},\left(\bigcap_{i \in l} B_{i}\right)$ $\bigcap\left(\operatorname{co}\left\{y_{i}: i \in l\right\}\right)=\phi$. For each $i=1, \ldots, n$, let $A_{i}=Y \backslash B_{i}$, then $A_{i}$ is a closed subset of $Y$. But then $c o\left\{y_{i}: i \in l\right\} \subset \bigcup_{i \in l} A_{i}$ for each non-empty subset $l$ of $\{1, \ldots, n\}$, so that by Theorem $7 \mathrm{C}, \bigcap_{i=1}^{n} A_{i} \neq \phi$. It follows that $\bigcup_{i=1}^{n} B_{i} \neq Y$ which is a contradiction. Therefore there exists a non-empty subset $\left\{i_{1}, \ldots, i_{k}\right\}$ of $\{1, \ldots, n\}$ such that

$$
\left(\bigcap_{j=1}^{k} B_{i_{j}}\right) \cap\left(\operatorname{co}\left\{y_{i_{j}}: j=1, \ldots, k\right\}\right) \neq \phi .
$$

\section{References}

[1] J. P. Aubin, "Mathematical Methods of Game and Economic Theory", North-Holland, Amsterdam, Revised Edition, 1982.

[2] J. P. Aubin and I. Ekeland, "Applied Nonlinear Analysis", John Wiley and Sons, New York, 1984.

[3] K. Fan, "Fixed-points and minimax theorems in locally convex topological linear spaces", Proc. Nat. Acad. Sci., U. S. A. 38 (1952), 121-126.

[4] K. Fan, "A minimax inequality and applications, in 'Inequalities III" ", Proceedings Third Symposium on Inequalities (Ed. O. Shisha), pp. 103-113, Academic Press, New York, 1972. 
[5] K. Fan, "Some properties of convex sets related to fixed point theorems", Math. Ann., 266 (1984), 519-537.

[6] I. L. Glicksberg, "A further generalization of the Kakutani fixed point theorem, with applications to Nash equilibrium points", Proc. Amer. Math. Soc., 3 (1952), 170-174.

[7] B. Halpern, "Fixed point theorems for set-valued maps in infinite dimensional spaces", Maith. Ann., 189 (1970), 87-98.

[8] S. Kakutani, "A generalization of Brouwer's fixed point theorem", Duke Math. J., 8 (1941), 457-459.

[9] B. Knaster, C. Kuratowski and S. Mazurkiewicz, "Ein Beweis des Fixpunktsatzes für n-dimensionale simplexe", Fund. Math. 14 (1929), 132-137.

[10] H. M. Ko and K. K. Tan, "A coincidence theorem with applications to minimax inequalities and fixed point theorems", Tarnkang J. Math., 17 (1986), 37-45.

[11] M. H. Shih and K. K. Tan, "Covering theorems of convex sets related to fixed point theorems", Nonlinear and Convex Analysis: Proceedings in Honor of Ky Fan, (Eds. B. L. Lin and S. Simons), pp. 235-244, Marcel Dekker, Inc., New York, 1987.

[12] M. H. Shih and K. K. Tan, "Shapley selections and covering theorems of simplexes", Nonlinear and Convex Analysis: Proceedings in Honor of Ky Fan, (Eds. B. L. Lin and S. Simons), pp. 245-251, Marcel Dekker, Inc., New York, 1987.

Department of Mathematics, National Central University, Chung-Li, Taiwan 32054, Republic of China.

Department of Mathematics, Statistics and Computing Science, Dalhousie Universityy, Halifax, Nova Scotia, B3H 3J5, Canada. 